\title{
Demographic Transition in Asia and its Consequences
}

\author{
Athar Hussain, Robert Cassen and Tim Dyson
}

\section{Introduction}

Over the five decades or so from 1950, Asia has witnessed a dramatic demographic transition, affecting the population growth rate, deaths and births. It has implications for the environment, schooling, the position of women and social security. The salient changes involved in this transition include:

- Reduction in the infant mortality rate by over two-thirds, from 184 infant deaths to 51 per 1,000 live births and an increase in life expectancy at birth by 25 years

- Decline in the total fertility rate (TFR) by more than half, from around six children per woman to 2.6

- Decrease of 0.6 percentage points in the population growth rate.

Although the transition has been under way across the length and breadth of Asia, its onset, speed and present status vary greatly between and within countries. As a result, the national demographic profiles, which were generally similar in the 1950s, now differ widely and divide into three distinct regional clusters (see Table 1).

In India the internal differences are as pronounced as those between countries. The major southern states, i.e. Andhra Pradesh, Karnataka, Kerala, Maharashtra and Tamil Nadu, have been, and remain, perhaps two decades ahead in their experience of the

demographic transition compared with the big, populous northern and eastern states, in particular, Bihar, Madhya Pradesh, Rajasthan and Uttar Pradesh (UP). It is especially in the densely settled, landlocked, poor northern states of Bihar and UP that death rates and reproductive behaviour have been the slowest to change. Internal differences also exist in China, but they are less prominent and the basic division is between rural and urban areas rather than between groups of provinces.

\subsection{Population growth}

The current population growth rate for Asia as a whole is 1.1 per cent per year, which appears low, but it comes on top of a much higher rate over the last 50 or so years. Given an already massive total population, it would translate by 2025 into an extra 757 million people, raising the total population from 3.6 billion in 2004 to 4.3 billion in 2025. The increase would not only be large in absolute numbers but also unevenly distributed across Asia's three regions. Looking back over the last half century, India's population rose almost three-fold from 361 million in 1951 to just over 1 billion in 2001, while the Chinese population slightly more than doubled in 47 years, from 594 million in 1953 to 1,266 million in 2000. The difference between the population growth rates of the two countries over the 20 years to 2025 could be wider still. The Indian population could increase by

\begin{tabular}{cl}
\hline Table 1 Regional clusters & \\
\hline Region & Salient features \\
\hline South Asia & High population growth rate and high fertility \\
South-east Asia & Moderate population growth rate and moderate fertility \\
East Asia & Low population growth rate and low fertility \\
\hline
\end{tabular}


Table 2 Regional population shares, 2004 and 2025 (\%)

\begin{tabular}{lll}
\hline Region & Population share (2004) & Population share (2025) \\
\hline South Asia & 42.3 & 46.2 \\
South-east Asia & 15.4 & 15.7 \\
East Asia & 42.4 & 38.1 \\
\hline
\end{tabular}

27 per cent, close to three times faster than the 10 per cent increase in the Chinese population. There is an inevitable degree of uncertainty about such a future projection of the population, which continues to rise the further one looks ahead. However, the broad features of Asia's population over the next 20-25 years are fairly predictable.

The population growth rates across Asia's three regions differ greatly, ranging from 0.6 per cent in East Asia, 1.3 per cent in South-east Asia to 1.7 per cent in South Asia (here South Asia includes Iran). Projected over the next 20 years, 65 per cent of the increase in population (492 out of 757 million) will be in South Asia, which by 2025 would account for close to half of the population of Asia (46.2 per cent). Associated with this is the almost certain prospect of India displacing China as the world's most populous country by 2050 if not earlier. The population shares of the three regions in 2025 would be different from that in 2004, as Table 2 shows.

The change in the shares is not only significant but also consequential. This is because the principal indicators of the demographic transition, such as the fertility rate and the population growth rate often go together with a particular configuration of socioeconomic characteristics, such as the average educational attainment, especially among girls, and the per capita income. For example, higher fertility and population growth rate are generally associated with a lower literacy rate or average educational attainment, especially among girls, and a lower life expectancy. An increase in the population share of the region lagging behind in the demographic transition has adverse implications for the configuration of socioeconomic characteristics.

This holds for a group of countries, such as Asia, or for constituent units of a country at different stages of the demographic transition. India provides an apt example of the latter, which is more relevant for policy than the former. Its four large northern states, referred to earlier, and Orissa in the east have the most poverty, and have the fastest population growth rate and the slowest gross domestic product (GDP) growth rate. In contrast, the states in the south and west have a low population growth rate, a relatively low incidence of poverty and high GDP growth rates. The five states in the north and east have more than half of India's poor today; 20 years from now, they will have three-quarters of India's poor and a much higher population share than at present, unless recent trends change. This growing social and economic divide between the states in the north and east, on the one hand, and the south and west on the other, poses the biggest challenge on the social and economic front.

China presents a similar example, albeit much less stark than the Indian one. Due to the combination of socioeconomic factors and a comparatively lighter birth control, rural areas have had a higher population growth rate than urban areas. As the rural population's average educational attainment are lower and its health status poorer than those of its urban counterpart, a rise in its share acts as a drag on the average educational and health status of the national population. An uneven demographic transition across constituent units of a country has a negative side to it and calls for a policy response to counteract the rising share of the lagging component. The appropriate policy response is, in the Indian case, to promote a faster development in the lagging states in the north and east and, in the Chinese case, to close the gap in the educational attainment and health status between the rural and the urban population.

\subsection{Decline in mortality}

During the period 1950-2004, mortality declined sharply in most countries of Asia and preceded the fertility decline, by several decades in many cases. In the 1960s Indian women were still having an average of around six births, despite a steady fall in mortality over preceding decades. The crude death rate (CDR) 
Table 3 Maternal mortality rates, 1985-2002 (per 100,000 live births)

\begin{tabular}{|c|c|c|c|c|c|c|c|}
\hline \multicolumn{2}{|c|}{ Very high: >400 } & \multicolumn{2}{|c|}{ High: 400-200 } & \multicolumn{2}{|c|}{ Moderately high: 200-50 } & \multicolumn{2}{|l|}{ Low: <50 } \\
\hline Nepal & 540 & Indonesia & 380 & Philippines & 170 & Iran & 37 \\
\hline India & 540 & Bangladesh & 380 & Vietnam & 95 & Thailand & 36 \\
\hline Pakistan & 530 & & & Sri Lanka & 92 & Malaysia & 30 \\
\hline Laos & 530 & & & China & 53 & RO Korea & 20 \\
\hline \multirow[t]{2}{*}{ Cambodia } & 440 & & & & & Japan & 8 \\
\hline & & & & & & Singapore & 6 \\
\hline
\end{tabular}

dropped by two-thirds from around 24 per 1,000 during the early 1950s to eight per 1,000 in 2004. Correlated with this, life expectancy at birth in the region, which in the 1950 s averaged a mere 41 years, had by 2004 risen by 25 years, with a regional breakdown of 28 years in East Asia, 24 in South-east Asia and 23 years in South Asia. In 1950, no country in Asia had a life expectancy of more than 75 years and only a few equalled or exceeded 60 years. Now only Afghanistan, Cambodia and Timor Leste have a life expectancy of less than 60 at birth and all three have suffered from a long period of civil-cum-international wars. In India, life expectancy for both males and females more than doubled in the 53 years from 1947 to the turn of the twenty-first century - a rise that represents the greatest single improvement in the conditions of life in modern India.

Notwithstanding these dramatic improvements, the gap between high and low mortality countries continues to be wide, and wider still in infant mortality. The 'under-five mortality' rate per 1,000 live births ranges from 89 in South Asia, 53 in South-east Asia to 39 in East Asia, and 10 per 1,000 or less in Hong Kong, Japan and Singapore. The mortality pattern for South Asia is distinctive with very high death rates among children (up to age 15) and the elderly, indicating high incidences of infectious and parasitic diseases, which can be easily avoided by means of basic medical care. The gender differences in mortality and life expectancy in the South Asia region stand out as being contrary to the usual pattern. The mortality rate for girls (aged $\mathbf{0}-5$ ) is higher than the corresponding rates for boys, which reflects the widespread preferential treatment of boys. Similarly, life expectancy for females is not higher than that for males, as it normally is, but either the same, as in Bangladesh, or shorter, as in India. However, Sri Lanka and Iran, also in South Asia, conform to the usual norm of longer life expectancy of females than of males, as do South-east and East Asia.

Many of the infectious diseases, which have almost disappeared from some countries, are still rife in the developing countries of Asia. A similar pattern obtains for maternal mortality, a rare event in some countries but a frequent hazard in many others. The maternal mortality rates across Asia are distributed as shown in Table 3.

Once again South Asia stands out with its very high maternal mortality rate, Bangladesh and, especially, Sri Lanka being exceptions. The huge differences in the maternal mortality rate and the low rates in lower middle economies such as Thailand and Malaysia would suggest that much of maternal mortality in the countries with rates more than 50 is avoidable, often with simple interventions.

Thus far, HIV/AIDS has been a much less important cause of death in Asia than in Africa. But deaths attributable to AIDS have been on the rise. The estimated number of HIV/AIDS cases is just over 1 million in China and 4 million in India. Although these constitute very small percentages of the respective populations of China and India, they nevertheless make up a substantial proportion of the world total of HIV/AIDS cases because of the huge population of the two countries. The estimates of the infected population in both countries are subject to a wide error margin and the actual number may be much higher than the reported total for two reasons: first, the surveillance network, notwithstanding recent improvements, is still in its development phase and second, potential cases may avoid diagnostic testing because of the fear of the consequences of being a known HIV carrier and the social stigma attached to the disease. 
Table 4 Total fertility rates, 2004

\begin{tabular}{llllll}
\hline High: $>\mathbf{4}$ & \multicolumn{3}{l}{ Medium: $\mathbf{2 - 4}$} & \multicolumn{2}{l}{ Low: <2 } \\
\hline Afghanistan & 6.7 & Indonesia & 2.3 & China & 1.8 \\
Bhutan & 4.9 & Brunei & 2.4 & Japan & 1.3 \\
Pakistan & 4.9 & Malaysia & 3.0 & RO Korea & 1.2 \\
Cambodia & 4.6 & Philippines & 3.1 & Singapore & 1.4 \\
Laos & 4.6 & India & 2.9 & Thailand & 1.7 \\
& & Nepal & 3.6 & Sri Lanka & 1.9 \\
& & Iran & 2.3 & Vietnam & 1.9 \\
\hline
\end{tabular}

In both countries, the vectors of infection are sufficiently efficacious to produce a serious pandemic within a short period even with the reported number of HIV/AIDS cases. As well as China and India, Vietnam and Cambodia also stand at a crossroads of the epidemiological map, i.e. depending on the coverage and effectiveness of preventive measures, within a small time span the infected population may snowball or stabilise and even shrink. Thailand provides a notable example of a country that has managed within a short period to slow down the spread of HIV/AIDS through preventive measures. Even under the worst scenario, HIV/AIDS would over the next 20 or so years have little effect on demographic statistics at the aggregate level, such as life expectancy. The principal impact would be to divert already scarce health resources away from a wide range of illnesses to the care and treatment of HIV/AIDS cases.

\subsection{Fertility}

With a delay following the downward trend in mortality, the total fertility rate in Asia has dropped sharply from six children in the 1950s to 2.4 births per woman in 2004. Almost all countries have experienced a decline but the extent of the decline, its time pattern and the current rates vary greatly across countries and inter-regional differences within some countries, such as India, can be as large as those between countries. Whereas mortality decline affects all age groups, a decline in fertility initially affects the number of children only and thus has a major impact on the age structure of the population.

Countries in Asia divide into three categories in terms of their current fertility rates: high, medium and low (see Table 4).
In terms of the TFRs, South-east Asia is the most heterogeneous region with member countries in all three columns in Table 4. In contrast, East Asia is the most homogeneous: bar Mongolia, every country in the region has a below-replacement TFR and the region as a whole is in the final stage of transition. Similarly, apart from Sri Lanka, every country in South Asia has a higher than replacement level TFR. This contrast ties in with the population growth rate in South Asia being almost three times that in East Asia, 1.7 per cent compared with 0.6 per cent.

Among countries with a below-replacement level TFR, Vietnam is of particular interest because, like Sri Lanka, it is a low-income economy and one that has achieved a dramatic fall in the TFR from very high 6.7 births per woman in 1970-5 to the current figure of 1.9. Iran stands out as a majority Muslim country with a low TFR of 2.3 and one that has achieved a significant reduction in fertility under a regime with a strong religious influence. The decline is due in part to its efficient health service delivery system and the recent but strong commitment on the part of the government to provide family planning services. Also of note is Pakistan, Iran's neighbour and a Muslim majority state, with a very high TFR of 4.9 births per woman and a population growth rate of 2.4 per cent per annum. With a current population of 157 million, Pakistan is likely to become the fourth most populous state in the world by 2025 .

By international standards, the fertility decline in China has been extraordinarily rapid with associated changes in the age structure of the population crowded into a short period which elsewhere have been spaced over a much longer period. The control on the number of births, generally known as 'the 
one-child policy', introduced in 1979 did not initiate the fertility decline but followed its onset by around 16 years in urban areas and ten years in rural areas. Here it is worth noting that India adopted family planning as an official policy in the late 1950s, around 20 years earlier than China. China's birth control policy has had two effects, first, to lower the rural TFR below the replacement rate in just over ten years and, second, to stop the urban TFR from rising above the replacement rate in the 1980s, with the start of economic reforms. As a result of the policy, the gap between the rural and urban fertility rates, which in 1981 was wide, has by 1996-8 narrowed down considerably. The main accomplishment of China's birth control policy, of which one child per couple is a component, has been to achieve an exceptionally low fertility rate in its rural population within a short period, thus hastening the fertility decline that was already under way.

Even though the total fertility rate since 1991 has been below the replacement rate of 2.1, the Chinese population is expected to keep on rising for some decades, driven by the inertia of above-replacement fertility rates until the recent past. The exact time path of the approach to a non-rising total will depend on the future course of the fertility rate. Given the government commitment to maintaining the stringent limit on births in the foreseeable future, this may be shorter than the 50 years it roughly takes for the population to stabilise at the replacement fertility rate.

Unlike in China, the regional variation in the TFRs in India is large and is likely to remain so in the foreseeable future. The average TFR in the southern states is 2.2 births per woman, close to the replacement level, and two among them, Kerala and Tamil Nadu, already have TFRs below replacement. In contrast, the northern states of Bihar, Madhya Pradesh, and Rajasthan and UP average a TFR of over four. In these states, the impact of high levels of fertility per woman on population growth will be further magnified by the comparatively large percentage of younger age groups in the population. By themselves, the former states of Bihar and UP (i.e. as they were constituted before the creation of the states of Jharkhand and Uttaranchal in 2000) may well contribute about 40 per cent of India's total population growth in the 25 years from 2001 to 2026. The population of the former UP could well be around 270 million by 2026 .
The differential demographic growth between the northern and southern states has implications for the human development indicators. These are generally better in the southern states, and the incidence of poverty, generally higher in the northern states. It could also contribute towards increased political tensions. Since 1977, political representation in the national Parliament has been frozen on the basis of the 1971 census results. However, the issue of correspondence between the numbers of people in each state and their political representation in Parliament is likely to come to the fore at some point, with potential for acrimony.

Turning to the issues arising from the demographic transition that Asian countries would face in the near future, these will vary with the stages of the transition. In the countries in the initial phase of the transition with a high TFR and population growth rate, such as Afghanistan, Cambodia, Laos, Nepal and Pakistan, the challenge would be to educate the swelling numbers of children in the 6-14 age group, and to provide employment to the entrants to the labour force. In the countries in the intermediate stage of demographic transition with a low TFR but with a comparatively high population growth rate due to the momentum of high TFRs in the immediate past (Bangladesh, Iran and Vietnam), some of the challenges will be the same as those facing the previous group of countries, e.g. educating the expanding number of children and coping with youth unemployment on top of an already substantial problem of adult employment. In addition, these countries will also increasingly face the implications of a low fertility on women's lives. The most important of these implications is the compression of life devoted to child bearing and rearing, thus widening the scope for the participation of women in the labour market. The increased importance of the non-reproductive role of women will bring into focus issues of gender disparity in, for example, educational attainment and the labour market outcomes.

For countries that have completed or will soon be completing their demographic transition, such as China, the Republic of Korea, Singapore and Sri Lanka, with a TFR below replacement and a low or negligible population growth rate, a major set of issues will be the ramifications of ageing population. Among this group of countries, Sri Lanka and China stand out as low-income economies with an already 
Table 5 Relation between TFR (2004) and age structure (\%)

\begin{tabular}{|c|c|c|c|c|c|c|c|c|}
\hline \multirow[t]{2}{*}{ High: >4 } & \multirow[b]{2}{*}{ Children } & \multirow[b]{2}{*}{ Elderly } & \multicolumn{3}{|c|}{ Medium: 2-4 } & \multirow[t]{2}{*}{ Low: <2 } & \multirow[b]{2}{*}{ Children } & \multirow[b]{2}{*}{ Elderly } \\
\hline & & & & Children & Elderly & & & \\
\hline Afghanistan & 43 & 3 & Indonesia & 29 & 5 & China & 23 & 7 \\
\hline Pakistan & 41 & 4 & Malaysia & 33 & 4 & Japan & 14 & 19 \\
\hline \multirow[t]{4}{*}{ Cambodia } & 41 & 3 & India & 33 & 5 & RO Korea & 20 & 8 \\
\hline & & & Nepal & 38 & 4 & Thailand & 23 & 7 \\
\hline & & & Iran & 31 & 5 & Sri Lanka & 24 & 7 \\
\hline & & & & & & Vietnam & 31 & 5 \\
\hline
\end{tabular}

large and a growing elderly population. Although not immediate, the problems arising from an ageing population also face the countries in the intermediate stage of transition. The ratio of the elderly in their populations is rising and will in some of the countries be substantial in the near future. Such countries include Vietnam, Thailand and in time also Bangladesh and Indonesia. As well as the looming issue of an ageing population, China also faces the problems of gender disparity in educational attainment and in the labour market. These assume special importance because China has one of the highest rates of participation of women in the labour market.

India is demographically too diverse to fit neatly into any one of the above three categories. Depending on the region, each of the categories is relevant. With their combination of a high TFR and a high population growth rate, the four northern states and Orissa in the east face the challenges of educating a growing population of children and of finding jobs for a rapidly expanding labour force. Southern and western states face issues that arise in the populations in the intermediate and final stages of the demographic transition. These include adapting to the implications of a low TFR for the socioeconomic role of women and addressing gender disadvantage in education and employment. In some of the southern states, the issues arising from an ageing population will soon loom large.

\section{Specific consequences}

\subsection{Age structure of the population}

The current and past TFRs have a far-reaching impact on the age structure of the population, usually summarised by the percentage of children (under age 15) and of the elderly (aged 65 or above) in the population. Table 5 shows these ratios for a sample of Asian countries classified by their TFR in 2004.

The countries with a high TFR - more than four children per woman - all have a percentage ratio of children of more than 40 . All three in the first column in Table 5 have a comparatively low literacy rate and face the formidable problem of schooling a huge and growing number of children. The countries with a medium TFR of between two and four children per woman receive a bonus in the form of a lower percentage of children and still low percentage of the elderly. Nepal has a high ratio because it had a high TFR in the recent past. The countries with a low TFR, below the replacement level, have, with the exception of Vietnam, a percentage ratio of children of less than 25 and a relatively high ratio of the elderly. The countries in this group have a lighter task of educating children but have to carry a heavier burden of supporting the elderly population. Compared with China, India has a heavier task of educating children, which is partially compensated by a lighter load of old age support.

The rising ratio of the elderly in the population in China has stimulated a widespread discussion of their financial support, which is currently divided between the family and the social security schemes. As increasingly realised in China, the current heavy reliance on the family for old-age support does not fit with the demographic trends and socioeconomic changes. The general trend is towards nuclear households and a majority of the elderly live separately from their offspring. With rising life expectancy, each succeeding cohort of the elderly would need support for a longer period than the preceding ones. Also, because of the birth control policy, which in many localities means one child per 
couple, the future cohorts of the elderly will have fewer offspring to depend on than the present cohort. The conjunction of birth control policy and the rising ratio of the elderly in the population has brought to the fore the issue of replacement of family financial support with old-age pensions. Currently, 62 per cent of the urban labour force is covered by the pension scheme under Social Insurance, which excludes the rural labour force. There are small-scale rural pension schemes but these cover only 54 million people (11 per cent of the rural labour force) and promise to provide only a percentage of the minimum subsistence. Although almost all of the discussion of pensions in China is focused on urban areas, the problem of the support of the elderly is more serious in rural than in urban areas. Not only do the rural elderly not receive a pension but also, given the trend of emigration of working-age adults from the countryside, the ratio of the elderly to working age adults will be higher in rural than in urban areas in the near future.

\subsection{Urbanisation}

Economic development involves population migration, mostly rural-to-urban and associated urbanisation, which both widens economic opportunities open to the labour force and creates problems such as the increased pressure on the environment. In Asia, as elsewhere, urbanisation has taken two forms: first, the expansion of existing urban settlements combined with rural-to-urban migration and, second, the urbanisation of rural localities without migration. The latter has been far more prevalent in China than in other developing economies and the driving force behind it has been the growth of rural industry. Reflecting the limited cultivable land area relative to the population, numerous rural counties in China have long had population densities similar to those in urban or periurban settlements. With rural industrialisation, many such counties have also come to derive most of their income from industry and services. But 'urbanisation without migration' has lost much of its force since the late 1990s with a sharp slowdown in the growth of employment in rural industry.

The reported urbanisation rates in India and China are 29 and 40 per cent, respectively. The designation 'urban' refers to a combination of high population density and the predominance of industry and services as sources of local income and employment. With continued population growth, much of rural
Asia has a high population density and faces issues characteristic of urban settlements, such as a heavy pressure of demand on the environment and on natural resources. As there is no universally agreed dividing line between 'urban' and 'rural', international comparison of the urbanisation rate is fraught with difficulties. By usual criteria in terms of population and the structure of the local economy, the urbanisation rate in China is significantly higher than the officially reported figure of 40 per cent because many rural counties are not only densely populated but also derive most of their income from nonfarming activities. One implication is that the difference in the urbanisation rate between India and China far exceeds the 11 percentage points based on the reported figures.

Raising the urbanisation rate has recently become a policy aim in China and the aim can be partly realised by a simple re-designation of already 'urbanised' rural counties. As in China, urban growth and urbanisation will continue in India. By 2026, about 35.6 per cent of the Indian population will be living in urban areas, i.e. a total of perhaps 506 million people. Much of southern India may experience comparatively little rural population growth. But much of northern India will experience considerable rural population growth and relatively rapid rates of urban growth too. There could well be an approximate doubling of the number of million+ cities by the year 2026, to 65-70 in total. And should the procedures used for classifying rural areas as urban somehow be relaxed, then all of these estimates could turn out to be too low. The two main foci for interstate migration are, and will remain, the urban agglomeration around Delhi, and the dynamic and increasingly integrated urban system of Maharashtra and Gujarat in the West. Our projections suggest that both Greater Mumbai and Delhi could be the centres of urban agglomerations containing almost 30 million people each by the year 2026. Indeed the UN estimates that as early as 2015, Mumbai and Delhi will have populations of 22.6 and 20.9 million, respectively, making them the second and third largest urban agglomerations in the world after Mexico City.

\subsection{Education}

The adult literacy rate varies widely between Asia's three regions. Universal or close to universal literacy is the prevalent norm in East Asia. At the other extreme is South Asia with a literacy rate that falls well short of 100 per cent. But intra-regional 
variation is much wider in South Asia, which includes subregions with universal literacy such as Kerala in south India and Sri Lanka. Focusing on India and China, the latter has always had a much higher literacy rate than the former. But the gap between the two has narrowed substantially thanks to an accelerated rise in literacy in India in recent years. Between 1991 and 2001, the overall literacy rate of the Indian population over seven years of age rose by 13 percentage points, to 65 per cent - the highest decadal rise ever. Even in the four large and educationally backward northern states, there was marked acceleration in the literacy rate among 10-14 year olds, which increased by 18 percentage points. Nevertheless there are huge educational backlogs throughout the country, with only modest numbers proceeding to secondary school and many tens of millions - especially in rural areas, and most particularly rural girls - receiving only rudimentary education or none at all.

There is a substantial population dimension to all this, past and future. In India, education is defined as a 'state' subject; major responsibility for educational provision rests with state governments. The large, poor northern states have only modest budgets for education, while the quality of public education is often dismally low, and their school-age populations have been growing rapidly. The school-age population of the big northern states, where education is relatively lacking, will still be growing 20 years from now; while in such states as Kerala and Tamil Nadu, where educational performance is currently better, the school-age population is already declining.

In China, the main problem is no longer raising the high literacy rate but achieving the target of nine years of basic schooling for all, which was set in 1986 to be fully implemented by 2000. Yet at the end of 2004, according to official statistics, close to 7 per cent of school leavers failed to reach the target. The actual shortfall may be wider than this. The shortfall would have been even larger but for the demographic bonus. Between 1982 and 2003, whereas China's population rose by 257 million (26 per cent), the number of school-age children fell by 12 per cent, or by 50 million from 243 to 193 million. This implies a reduction in the cost of achieving universal basic education, given the number of years of schooling. What this suggests is that the demographic factors have facilitated the achievement of the target of universal basic education rather than impeding it. The failure in reaching the target has been due to the lack of importance given to basic education in the past, which has been reversed over the last few years.

\subsection{Environment}

Asian countries face a wide range of environmental problems. Leaving aside those concerning biodiversity, they fall under two headings: pollution and the depletion of natural resources. In urban conurbations and in urbanised rural localities, ambient concentrations of hazardous particles and gases are many times over the safety limits. The contamination of surface and ground water is widespread and some regions of Asia, including the northern half of China, are critically short of water. Land degradation due to the discharge of solid wastes and hazardous matter is common. Soil erosion and deforestation in some localities have reached serious proportions. In one form or another, a large and growing percentage of population suffers the adverse impact of environmental pollution, especially of water. There are similarities between current problems of environmental pollution in developing countries of Asia, such as China and India, and also those in, for example, Japan and South Korea in the past, when environmental regulation was weak. However, given their huge populations, China and India's environmental problems are comparatively much larger in scale.

The influences of population growth on the environment are complex; but analysis suggests two main aspects: those related to pollutants from energy, industry and transport, and those related to water. The former are more strongly related to economic growth than to population increase, and are in the main susceptible to technological remedies; the latter is strongly related to population growth, and technology does not appear to offer many near-term remedies. China is responsible for 14 per cent of carbon emissions and India accounts for 5 per cent. In absolute terms both countries are among the top six countries for global emissions, even though in per capita terms their contribution is very low. With coal as the main source of energy and the rapid growth of motorised vehicles, both China and India are likely to grow into polluting giants unless effective environmental regulation is put in place. The growth in atmospheric pollution in both countries is mainly due to industry, energy and transport, which in both countries are growing 
rather faster than the population. It is economic growth, and the pattern of that growth, that is doing the damage, with population providing a background demand factor.

The demand for water is strongly related to population growth - not mainly for personal and residential use, which, in India, only takes some 5 per cent of fresh water supplies, but for food and agriculture, which takes some 80 per cent of supplies. The pattern of the demand for water in China is broadly similar to that in India, except that the percentage share of agriculture is lower and that of industry higher. Both countries aim to remain

\section{Bibliography}

Chopra, K. and Goldar, B. (2000) Sustainable Development Framework for India: The Case of Water Resources, final report for the United Nations University, 'Tokyo Project on Sustainable Development Framework for India', Delhi: Institute of Economic Growth

Dyson, T., Cassen, R. and Visaria, L. (2004) TwentyFirst Century India - Population, Economy, Human Development, and the Environment, Oxford: Oxford University Press

Gubhaju, B. and Moriki-Durand, T. (2003) 'Fertility Levels and Trends in the Asian and Pacific Region, Ch. 2, Population and Development, Asian Population Studies Series 161

Hussain, A. (2002) 'Demographic Transition in China and its Implications', World Development 30: 10

IUP/IHD (2000) India Water Vision 2025: Report of the Vision Development Consultation, New Delhi: India Water Partnership/Institute for Human Development largely self-sufficient in food, which will require an increase in the demand for water in line with any increase in population. As extra supplies of water are limited or negligible, much of the additional demand has to be met by raising efficiency in the use of water and increased reliance on recycled water. In both countries, investment in waste water treatment falls well short of what is needed and the regulatory framework for promoting efficiency is still not in place. As a result, water shortage, especially of fresh water, is likely to be a ubiquitous problem in India and China, as well as in the rest of developing economies of Asia.

Mari Bhat, P.N. (2002) 'India's Changing Dates with Replacement Fertility: A Review of Recent Fertility Trends and Future Prospects', in Completing the Fertility Transition, New York: United Nations Department of Social and Economic Affairs Population Division, ESA/P/WP.172/Rev.1

Seetharam, K.S. (2002) 'Half a Century of Unparalleled Demographic Change: The Asia-Pacific Experience', Asia-Pacific Population Journal, December

UNICEF (2005) The State of the World's Children 2005, New York: United Nations Children's Fund

United Nations (2005) World Population Prospects: The 2004 Revision Population Database, New York: United Nations

Working Group on Perspective of Water Requirement (WG) (1999) Report of the Working Group on Perspective of Water Requirements (National Commission on Integrated Water Resources Development Plan), New Delhi: Ministry of Water Resources, Government of India 